
25 Research Square
Preprints are preliminary reports that have not undergone peer review.
They should not be considered conclusive, used to inform clinical practice,
or referenced by the media as validated information.

\title{
Dental Pedagogy in the 'New Normal' COVID-19 Era: A Transition Template of Teaching Protocols
}

Nadia Khalifa

University of Sharjah

Lakshman Perera Samaranayake ( $\square$ lakshman@hku.hk)

The University of Hong Kong, Hong Kong Special Administrative Region

Kausar Sadia Fakhruddin

University of Sharjah

\section{Research Article}

Keywords:

Posted Date: February 3rd, 2022

DOI: https://doi.org/10.21203/rs.3.rs-1036301/v1

License: () (i) This work is licensed under a Creative Commons Attribution 4.0 International License. Read Full License 


\section{Abstract \\ Background}

Covid-19 is a virus known for weaseling out of preconceived notions and precautions, increasing the dire need for combative measures to match. However, SARS-CoV-2 continuously poses fundamental problems to dental personnel owing to work in close immediacy to the patient during treatment and aerosolgenerating procedures (AGPs). Resuming routine clinical operations, especially where students are the care providers in the teaching facility, is a daunting challenge. In connection with this pandemic, general practice dentists need modified operating procedure (MOP) guidelines.

\section{Methods}

In the absence of unified guidelines for modified infection control/clinical procedures for dental education, we implemented a clinical protocol template and MOP for teaching clinical dentistry to fit the infection control requirements during the pandemic/post-pandemic period at the University of Sharjah, College of Dentistry, UAE.

\section{Results}

These were based on engineering control measures (e.g., negative-pressure ventilation systems in operatories) to administrative control measures, which included fallow periods of treatment-abeyance between patient sessions after procedures. The consequent extension of teaching periods and clinical sessions is a thorny scenario for schools with hundreds of students and minimal teaching and support staff.

\section{Conclusions}

A new method of operating protocols that complements the existing operating protocols in clinical dentistry aims to eliminate nosocomial infection transmission and protect patients and care providers. These proposals and the templates provided are unavoidably interim measures, given how the pandemic is evolving. Protocol reviews should be conducted whenever new information about COVID-19 and its spread emerges.

\section{Background}

World Health Organization (WHO), in January 2020, announced a new human pneumonia-like outbreak in Wuhan, China, termed the coronavirus disease 2019 (COVID-19), caused by the severe acute respiratory syndrome coronavirus 2 (SARS-CoV-2) infection, now a global pandemic of unprecedented proportions. The ravaging impact of SARS-CoV-2 is profound, with the global population under 'lockdown' conditions in several countries, struggling to contain the pandemic's recurrent waves [1]. In addition, there is now clear evidence to demonstrate SARS-CoV-2 transmission from person to person through many vectors, such as aerosols, respiratory droplets, and contact spread [2,3] into unsuspecting victims via entry portals such as the nose, mouth, and eyes [4].

The major impediment for reining in the COVID-19 pandemic is the high prevalence of up to $40 \%$ asymptomatic disease carriers in the community. Radical containment measures to limit disease transmission have included social distancing, thorough hand washing, quarantine, and the use of personal protective equipment such as masks [5]. In addition, the advent of the vaccines for COVID-19 and rapid diagnostic, such as LAMP tests [6], are other preventive measures that are eagerly awaited in this context $[5,7]$.

Compared to the general population at large, healthcare workers (HCWs), including dental professionals, run a greater risk of contracting respiratory pathogens by nature of their profession. This was evident in the SARS epidemic of 2013, which led to numerous deaths of HCWs [8] and in the current COVID19 pandemic, where HCWs are disproportionately affected [9]. Thankfully, there has been no documented mortality amongst dental healthcare workers (DHCWs) due to COVID-19. However, they are the professional group that is thought to have the highest likelihood of acquiring the infection in comparison to other clinical professions [10], according to the World Health Organization (WHO) [11].

It is clear from the incessantly unfolding pandemic scenario that the disease will be entrenched in the global community for the foreseeable future until an effective and efficacious vaccine is found, in addition to therapeutic interventions that reduce morbidity and mortality. In such an adverse climate, dental educators need to rethink and reinvent effective and efficient delivery modes of clinical dental education. As opposed to clinical medicine, the latter is particularly challenging in this context due to the inevitable and obligatory aerosol-generating procedures (AGPs) used for dental patient management while working for prolonged periods in close proximity to patients who may be unknowingly harboring the SARS-CoV-2. For instance, it is well known that in dentistry, the routine use of high-speed handpieces with water jets, air/water syringes, ultrasonic scaling, and air polishing cause such aerosolization of microbes into the ambient clinic air [12].

\section{Dentistry In A Teaching Clinic In Uae}

After the pronouncement by the World Health Organization that SARS-CoV-2 infection is a pandemic, on 11th March 2020, the Ministry of Health and Prevention, in liaison with the Ministry of Education of the United Arab Emirates (UAE), advised cessation of clinical dentistry, apart from urgent procedures, in all primary care dental practices in the country. The university dental colleges followed these instructions in UAE, and all non-clinical teaching was moved to virtual platforms. Furthermore, university examinations were rescheduled and remodeled, and criteria for the successful graduation of students were reviewed and modified considering the prevailing conditions. 
This situation persisted until mid-July 2020, after which a decision was taken by UAE authorities for the resumption of dental care delivery in accordance with stringent guidelines provided by international and national health regulatory bodies [13]. The post-COVID-19, New Operational Model (NOM), which

supplemented Standard Operating Procedures in clinical dentistry, was an attempt to mitigate infection transmission to four different groups of stakeholders, namely i) the patient, ii) the dental student, iii) the clinical academic, and iv) the para-dental personnel/assistants.

In the absence of standard universally promulgated guidelines on obviating COVID-19 nosocomial transmission in the dental educational environment, we provide below salient features of a New Operational Model (NOM) for a dental hospital teaching facility in UAE. These guidelines could be used to offer protection to all stakeholders while simultaneously delivering safe and efficacious clinical care for the patient and uninterrupted education for aspiring dentists. The latter group is doubly unfortunate as they are not only exposed to a vicious infection but also to its secondary effects that have impacted their professional prospects and future livelihood.

\section{The Profile Of The Dental Teaching Hospital}

University Dental Hospital Sharjah-UAE (UDHS-UAE) is a primary dental healthcare facility equipped with 124 dental units. It is home to more than 125 academic and non-academic staff and approximately 186-undergraduate clinical students. During the pre-COVID era, the Hospital provided comprehensive dental care to some 150 patients daily, during two clinical sessions of 3 hours each. Using a curriculum based on the comprehensive dental care delivery teaching model, the undergraduate trainees and residents are mentored to care for all walk-in patients irrespective of their socio-economic status.

\section{Mitigation Of Nosocomial Infection During Covid-19 (Figure 1)}

In considering infection control in an institutionalized setting or otherwise, the analytical and the subsequent decision-making processes should, in general, be based on the following three main principles.

3.1. Administrative controls which offer work policies and procedures to reduce the exposure of all stakeholders to the identified hazard and change the way people work.

3.2. Engineering controls entail changes in the physical features of the workplace, such as hospital design and configuration, to mitigate pathogen exposure at the source and improve compliance.

3.3. Personal protective equipment (PPE) utilization that needs to be considered when both the engineering solutions and administrative controls are deemed insufficient. (Note: PPE are not described here as it is beyond the remit of this article)

The following describes in detail how the MOPSs entailing engineering and administrative control measures were planned at the University Dental Hospital Sharjah, UAE (UDHS-UAE) (Figure 2)

\subsection{Administrative controls}

The administrative controls were sub-categorized as a) general measures and b) those appertaining to work practices. These are outlined below.

\subsubsection{Administrative controls - General measures}

\subsection{1a. Modified clinic settings (physical changes at the workplace) and temporality of care delivery}

The UDHS-UAE dental clinic's architectural profile is the standard model, featuring multiple, partially isolated cubicles set in clusters and rows, with separate clinics devoted to specialized care. The new guidelines for infection mitigation entailed alternate color-coding of each cubicle, either as blue or yellow, with 18blue coded functioning units alternating with an equivalent number of yellow coded units (Figure 3 ). To maintain the social distancing ethos, this initiative would ensure that only a single-color coded clinic, of either blue or yellow, was operational at a given time, with no adjacent cubicles used for simultaneous patient care.

As a consequence of this administrative control, the clinic's functional capacity is reduced by 50 percent, leading to knock-on issues that entail longer teaching hours extending well into the late evening and modified, sub-divided, multiple clinical sessions for each student group. In practical terms, the blue/yellow coded cubicle arrangement permits a more than 4-meter space between two functioning units, thus maintaining a safe, social distancing system to obviate infection transmission. This arrangement also means that the alternate cubicles can be deep cleansed with the increased fallow time between patient sessions (see below).

\subsection{1b. Student subgrouping to facilitate social distancing.}

During the pre-COVID era, the students of BDS years four and five were paired together (the buddy system) to deliver clinical care guided by four-handed dentistry philosophy from 9 am to 4 pm. However, the new teaching arrangements of split teaching sessions and stretched teaching periods and clinical sessions well into the evening hours ( 8 am to $8 \mathrm{pm}$ ) necessitated an increase in manpower such as general practitioners and additional specialists from different dental specialties assigned to work during each clinic session, Figure 5.

\subsection{1c. Pre-clinic COVID-19-check of DHCWs and students}

In the UAE, the government has mandated a contact tracing application, ALHOSN, which utilizes blue-tooth technology to swiftly monitor and alert individuals of their COVID-19 health status [14]. This is accomplished through a QR-based color-coded system that is verifiable and can be scanned and refreshed every two minutes. 
A unique QR code may comprise one of four colors (grey, green, red, or amber). An untested individual displays a 'grey' QR-code. In contrast, a negatively tested or healthy individual with a 'green' code grants the individual access to all public spaces. A 'red' QR code, on the contrary, signifies a 'COVID-positive' individual. An 'amber' QR code implies a test or re-test for possible COVID-19 exposure. Accordingly, only the 'green' QR-coded faculty members, administrative and clinical staff, students, and resident dentists are eligible to enter the hospital premises after they are temperature scanned at the Hospital's designated, dedicated entry point.

Besides temperature checks, hand sanitization at the designated entrance for DHCWs, teachers, and students is required. In addition, all DHCWs are mandated to don full enhanced personal protective equipment (PPE), including an N95 respirator, protective eyewear, face shield, disposable gowns, and protective layering on footwear in designated PPE donning areas (see below).

\subsection{1d. Modified patient screening and triage protocol:}

Patient entrance to the Hospital is organized via a designated entry point after passing through the installed 'sanitizing tunnel,' leading to a 'fever check' booth. Following hand sanitization and fever check, the designated resident doctor in full enhanced PPE records COVID-19 related symptoms (if any) from the patients in a 'patient triage questionnaire.' Only patients showing apparent COVID-negative symptoms are allowed in the UDHS - Comprehensive Care Clinics.

In the event of a COVID-19 suspect or positive patient being identified, emergency care is provided. The designated nurse escorts such patients to the negative pressure, 'isolation clinic' dedicated for emergency dental procedures. Furthermore, a suspected COVID-19 case with non-emergency dental needs is referred to the adjoining, designated Emergency Care Department of the Medical Facility of the Sharjah University Hospital, which has special facilities that receive suspect-COVID-19 patients.

\subsection{1d. Communal social Distancing measures}

The communal seating in the waiting area is arranged to permit a social distance of 6 -feet between two seated patients [11]. Additionally, only a single essential visitor accompanying the patient is allowed within the hospital premises, either in the communal waiting area or within the clinical bay, for necessary assistance during the patient treatment session. Likewise, faculty, staff, students are only permitted into hospital premises when scheduled. Upon patient discharge from the clinic, the patient, and the attendee, if any, are escorted out of the hospital premises immediately afterward by an assigned staff member.

\subsubsection{Administrative controls related to Work Practices:}

\subsection{2a. Modified scheduled for AGP without dental dam:}

Where possible, students are encouraged to avoid AGPs, using hand excavators for carious dentine excavation and hand instruments for scaling and root planing. The use of a rubber dam is essential. If dental procedures such as ultrasonic scaling, subgingival restorations, or surgical procedures that are generating copious bioaerosols cannot be avoided and rubber dam utilization is not possible [15-17], then these specific treatments should be scheduled towards the end of the session to allow for a fallow period of treatment abeyance.

\subsection{2b. Designated PPE donning and doffing areas}

Wearing (donning) personal protective equipment (PPE) before a clinical session and removing (doffing) them afterward should be done with extreme care as the newly donned PPE should not be contaminated. In contrast, in the post-clinic session, contaminated PPE should be discarded not to contaminate any other source with which they come into contact. Hence separate donning areas (clean areas) and doffing areas (dirty areas) should be designated in any dental clinic. This situation gets further complicated when numerous students and teaching and para-dental support staff work in a multi-unit teaching clinic. Hence, distinct designated areas for 'donning' and 'doffing' of personal protective equipment (PPE) must be created in each teaching clinic. It is critical to have either adequate natural or negative pressure ventilation in such areas that should be large enough to accommodate social distancing.

\subsection{2c. Designation of fallow periods for clinics}

A period of treatment abeyance between two different patient sessions in a clinical unit is termed the fallow period. The fallow period needs to be determined and guided by the clinical procedure undertaken, as well as the use of AGP and the ambient ventilation. UK dental authorities have recently provided extensive guidance on determining and calculating the fallow period [18]. For instance, a clinical procedure using a rubber dam, AGP, and HEPA filtration evacuation should have a $45 \mathrm{~min}$ fallow period [19]. The designated fallow periods in our teaching clinics are 3 hours because of the alternate use of working cubicles.

\subsection{2d. Deep cleaning of the dental operatory}

Following the fallow period, the assigned nursing team dons appropriate PPE and disinfects operatories with particular attention to high-contact surfaces, utilizing appropriate hospital-grade disinfectants (Viruton ${ }^{8}$ Forte, MEDISEPT) prior to the arrival of the next patient.

\subsection{2e. Removal of potential fomites}

All unnecessary shelving, furniture, brochures, pamphlets, and magazines that may surreptitiously act as fomites for vector transmission of infection are removed from the clinical areas and the hospital lobby. These inanimate objects or fomites frequently handled by clinic attendees and staff cannot be easily sanitized and are known to be potential vectors of viral transmission [11].

\subsection{2f. Diagnostic Imaging}


Current recommendations from local and international regulatory bodies are to defer or avoid intraoral radiographs as much as possible during the pandemic. Such procedures frequently evoke coughing or gagging reflex in the patient, thereby generating microbe-laden aerosols [20, 21]. Hence, intraoral procedures in the imaging units are replaced by extraoral bitewings, extraoral lateral oblique view, sectional or full-width dental panoramic (OPG) radiography as alternative imaging techniques [21] whenever possible.

\subsection{Engineering controls}

Many interventional procedures are known to aerosolize respiratory secretions in healthcare settings [12, 22]. In dentistry, viral particles may be aerosolized by the high-speed handpiece and the accompanying air jet, ultrasonic scaling, air polishing, and air/water syringes. To reduce bioaerosol generation, especially in settings where multiple patient treatment sessions are simultaneously conducted [12], as in dental teaching hospitals, the following control measures are necessary i) extra-oral high-volume evacuation, ii) negative pressure ventilation, iii) air filtration, iv) ultraviolet irradiation of the operatories v) plastic barriers in patient-administrator communication portals vi) red-green lighting system in each cubicle to indicate the use of AGPs [23,24].

\section{2a. High-volume evacuators (integral and portable)}

Air purification systems to ease bio-aerosol concentrations in the operatories while the patient is undertaking AGP, in addition to the retrofitted filters, which are integral components of the hospital air conditioning system [25], are essential. These additional portables, HVE, can further mitigate the burden of aerosolization of bio-contaminants stemming from the operative site.

\section{2b. Air filtration}

Additionally, to decontaminate the clinics' ambient exhaust air, a two-layered air filtration system using high-efficiency particulate air (HEPA) filters are necessary [11]. These can be incorporated and retrofitted into the currently extant centralized heating, ventilation, and air-conditioning (HVAC) system. These HEPA filters with MERV-13 level (Minimum Efficiency Reporting Values) efficiency levels mechanically trap particles on impact and capture 99.97\% of 0.3micron size contaminants that pass through the filters [26].

\section{2c. Negative pressure ambiance}

Rooms with negative air pressure help keep airborne viruses and other microbes from entering the surrounding rooms and hallways, thus decreasing the potential risk of COVID-19 spread from clinics [27]. Hence, in addition to the air evacuation systems described above, all the teaching clinics should be retrofitted and maintained with a negative pressure ventilation system throughout the Hospital. Previously, only isolation rooms had a negative pressure ventilation system.

\section{2d. Ultraviolet irradiation}

Ultraviolet (UV) germicidal irradiation utilizes short wavelength UV light to inactivate microbes, including viruses, essentially disrupting their nuclear DNA/RNA. They are generally harmless for humans unless on exposure for prolonged periods. Therefore, an additional engineering control measure is the installation of upper-room ultraviolet germicidal irradiation (UVGI) units. This environmental infection control measure, once operative overnight (for 18 hrs), when there is no clinical activity, is known to inactivate microbes in the operatory ambient air, adjacent hallways, and patient waiting areas of the Hospital [28].

\section{2e. Plastic barriers in (patient-administrator) communication portals}

Further engineering measures to restrict close contact between administrative staff and patients in the reception areas include installing a transparent plastic/glass barrier between the receptionist and the patient with a communication portal.

\section{2f. Indicator lighting system}

Another ancillary engineering control measure is an indicator lighting system in each cubicle where a green light implied a non-AGPs, and a red light implied AGPs being in current use.

\section{2i. Occupational health-risk awareness measures}

Last but not least, behavioral changes of the dental health care workers (DHCWs), including students, are a prerequisite for the proper execution of infection control measures [29]. Awareness of updated infection control methods related to occupational health risks through the 'webinar' series needs to be organized for dental staff and students on a regular basis. Additionally, infographics displaying appropriate steps for donning and doffing PPE should be displayed in the assigned areas. Furthermore, infographics and posters on the pandemic's current status need to be regularly posted in all appropriate areas of the Hospital and displayed on the television screens in the patient waiting areas.

\section{Logistic management during COVID-19 pandemic:}

While many dentistry colleges across the globe chose to close the clinics and delay student graduations due to the pandemic, ours did not. Alternatively, efforts were invested in continuing the clinical and pre-clinical training sessions for our students in accordance with the constraints. Abiding by social distancing practices, students were distributed into subgroups, with labs and clinical sessions spread out, which stretched to increased hours. Supervision was further individualized due to student subgrouping. Therefore, hiring additional part-time staff was prioritized, so overall competency requirements were not compromised. 
In consideration of reduced patient flow consequent to the pandemic, clinical requirements were adjusted with the inclusion of case-based scenarios performed on dummies in clinics. As a result, additional lab and clinical manuals were prepared by faculty, seen illustrated in Figure 4.

Furthermore, as illustrated in the timeline in figure 6, exams and classes were resumed on time without delay. Even upon the arrival of the vaccines, which are available within the university premises, PCR tests remain mandatory for staff and students alike. This has remained especially so in consideration with variant risk as visualized in Figure 6.

Students who contracted COVID were not marked as absent and were provided extra resources to complete their requirements missed during the quarantine period. In addition, the off-site examination was arranged for them with additional invigilation staff for the task.

The dynamic scenario of the recent global pandemic has allowed the implementation of the hybrid model followed by our hospital. Our logistic policies have been influenced and guided by the actions taken by other health regulatory bodies and institutions globally [30-32].

\section{Conclusions}

COVID-19 pandemic is continuing unabated in most regions of the world. The $\neq w\|a\| l$ implies that the community as a whole need to live and conduct their daily business in the presence of this vicious virus foe, well into the foreseeable future until the advent of efficacious vaccines, therapeutic and rapid diagnostics. Therefore, a correct balance needs to be struck to offer students the necessary training while not compromising on the required infection control measures needed for the safety of students, faculty, and patients. Hence the structure of the new modified operative procedures (MOP) for the University Dental Teaching Hospital Sharjah, described in detail here, should serve all stakeholders in the dental community and the public at large to mitigate infection spread until the pandemic abates. Logistic management is definitely a challenge and requires the implementation of adaptation. Our MOP explains how the challenges we face were addressed. The many arms of the administrative and engineering controls that need the implementation to prevent nosocomial infection spread could be instigated in similar dental teaching hospitals in other jurisdictions. However, the financial resources required for engineering controls could be substantial and burdensome, though the administrative controls may not require such outlays. Under these circumstances, an adopt and adapt attitude may have to be taken by relevant authorities considering clinical dental teaching in the new normal era.

\section{Abbreviations}

\section{MOP}

Modified Operative Procedure

UVGI

Ultraviolet Germicidal Irradiation

HEPA

High-Efficiency Particulate Air

AGP

Aerosol-Generating Procedure

\section{Declarations}

\section{Ethics approval and consent to participate}

Not applicable.

\section{Consent for publication}

All contributing authors consented to publication

\section{Availability of data and material}

Not Applicable, as this is a report on the management strategies adopted during the current COVID-19 pandemic in the Dental Educational Establishment. However, if required, the corresponding author can provide data included in the present study on reasonable request.

\section{Funding}

This work was funded by the University of Sharjah oral biofilm in health and disease group establishment fund.

\section{Author Contributions}

KSF and NK performed data collation analysis and manuscript writing; LPS critically examined and revised the manuscript. All authors approved the final version to be published.

\section{Acknowledgment}

I would like to thank Dr. Amel Hamzeh, Dr. Abier Abdul Sattar, and Dr. Mohamed El-Kishawi for their organizational inputs. 
${ }^{1}$ Department of Preventive and Restorative Dentistry, University of Sharjah, UAE. ${ }^{2}$ Faculty of Dentistry, University of Hong Kong, 34 Hospital Road, Hong Kong.

\section{References}

1. United Nation Development Program UNDP: Putting the UN framework for socio economic response to COVID-19 into action. INSIGHT, United Nation Development Program (UNDP). https://reliefweb.int/report/world/brief-2-putting-un-framework-socio-economic-response-covid-19-action-insights-june2020. June 2020, Brief \#2:1-19.

2. World Health Organization WHO: Clinical Management of COVID-19- Interim guidance, 27 May 2020, World Health Organization (WHO). https://apps.who.int/iris/handle/10665/332196. 2020:1-62.

3. Peng X, Xu X, Li Y, Cheng L, Zhou X, Ren B: Transmission routes of 2019-nCoV and controls in dental practice. International journal of oral science 2020, 12(1):9-9.

4. Samaranayake LP, Fakhruddin KS, Panduwawala C: Sudden onset, acute loss of taste and smell in coronavirus disease 2019 (COVID-19): a systematic review. Acta Odontologica Scandinavica 2020:1-7.

5. Chu DK, AkI EA, Duda S, Solo K, Yaacoub S, Schünemann HJ, Chu DK, Akl EA, El-harakeh A, Bognanni A et al: Physical distancing, face masks, and eye protection to prevent person-to-person transmission of SARS-CoV-2 and COVID-19: a systematic review and meta-analysis. The Lancet 2020, 395(10242):1973-1987.

6. Samaranayake LP, Kinariwala N, Perera RAPM: Coronavirus Disease 2019 (COVID-19) Diagnostics- a Primer. Dental Update 2020, 47:761-765.

7. Nishiga M, Wang DW, Han Y, Lewis DB, Wu JC: COVID-19 and cardiovascular disease: from basic mechanisms to clinical perspectives. Nature Reviews Cardiology 2020.

8. Samaranayake LP, Peiris M: Severe acute respiratory syndrome and dentistry: a retrospective view. Journal of the American Dental Association (1939) 2004, 135(9):1292-1302.

9. Wang J, Zhou M, Liu F: Reasons for healthcare workers becoming infected with novel coronavirus disease 2019 (COVID-19) in China. The Journal of hospital infection 2020, 105(1):100-101.

10. Jamal M, Shah M, Almarzooqi SH, Aber H, Khawaja S, El Abed R, Alkhatib Z, Samaranayake LP: Overview of transnational recommendations for CoVID19 transmission control in dental care settings. Oral Diseases 2020.

11. World Health Organization WHO: Consideration for the provision of essential and oral health services in the context of COVID-19. Interim Guidance. 3rd August 2020. 2020:1-5.

12. Coulthard P: Dentistry and coronavirus (COVID-19) - moral decision-making. British Dental Journal 2020, 228(7):503-505.

13. Emirates MoH-UA: MOH-Guidelines-for-re-opening- 5 June, 2020. 2020:1-29.

14. Ministry of Health MOH: ALHOSN APP -UAE.pdf, Ministry of Health (MOH), United Arab Emirates. https://www.ncema.gov.ae/alhosn/index.html. 2020:1-9.

15. Cochran MA, Miller $\mathrm{CH}$, Sheldrake MA: The efficacy of the rubber dam as a barrier to the spread of microorganisms during dental treatment. $J$ Am Dent Assoc 1989, 119(1):141-144.

16. Timmerman MF, Menso L, Steinfort J, van Winkelhoff AJ, van der Weijden GA: Atmospheric contamination during ultrasonic scaling. J Clin Periodontol 2004, 31(6):458-462.

17. Ishihama K, lida S, Koizumi H, Wada T, Adachi T, Isomura-Tanaka E, Yamanishi T, Enomoto A, Kogo M: High incidence of blood exposure due to imperceptible contaminated splatters during oral surgery. Journal of oral and maxillofacial surgery 2008, 66(4):704-710.

18. Samaranayake L: Dental Practice during the COVID17 Pandemic-Two Pathfinder Documents Point the Way. Dental Update 2020, 47:761-765.

19. Clarkson J, Ramsay C, Richards D, Robertson C, Aceves-Martins M: Aerosol Generating Procedures and their Mitigation in International Dental Guidance Documents - A Rapid Review. https://oralhealth.cochrane.org/news/aerosol-generating-procedures-and-their-mitigation-internationalguidancedocuments. 2020:1-69.

20. Hamedani S, Farshidfar N: The practice of oral and maxillofacial radiology during COVID-19 outbreak. Oral radiology 2020:1-4.

21. Little R, Howell J, Nixon P: COVID-19 and beyond: implications for dental radiography. British Dental Journal 2020, 229(2):105-109.

22. Harrel SK, Molinari J: Aerosols and splatter in dentistry: A brief review of the literature and infection control implications. The Journal of the American Dental Association 2004, 135(4):429-437.

23. Li RWK, Leung KWC, Sun FCS, Samaranayake LP: Severe Acute Respiratory Syndrome (SARS) and the GDP. Part II: Implications for GDPs. British Dental Journal 2004, 197(3):130-134.

24. Health MDo: Airborne Infectious Disease Management. Methods for temporary negative pressure isolation. . 2007:1-41.

25. Dubai Health Authority DHA: Health Facility Guidelines 2012 for Planning, Design, Construction and Commissioning.

https://www.dha.gov.ae/Documents/Regulations/Guidelines/Health\%20Facility\%20Guidelines\%20_Planning\%20Design\%20Construction\%20and\%20Cor 2012, 00:1-59.

26. Aerosols Al: ASHRAE Position Document on Infectious Aerosols.

https://www.ashrae.org/file\%20library/about/position\%20documents/pd_infectiousaerosols_2020.pdf. . April 14 2020:1-24.

27. Anderson J, Geeslin A, Streifel A: Airborne Infectious Disease Management, Methods for Temporary Negative Pressure Isolation. Office of Emergency Preparedness, Minnesota Department of Health. . 2007:1-41.

28. HANDBOOK A: Ultraviolet air and surface treatment, chapter 62. ASHRAE handbook -HVAC Applications (SI). https://www.ashrae.org/file\%20library/technical\%20resources/covid-19/si_a19_ch62uvairandsurfacetreatment.pdf. 2019:1-18. 
29. Blignaut E, Nemutandani S, Samaranayake L: Strategic implementation of dental infection control in resource-poor jurisdictions during CoVID-19 pandemic: a perspective from South Africa. Acta Odontol Scand 2020:1-4.

30. National Health Service NHS: Scottish Dental Clinical Effectiveness Program -Resuming General Dental Services Following CoVID-19 Shutdown Update. https://www.sdcep.org.uk/wp-content/uploads/2020/06/SDCEP-Resuming-General-Dental-Services-Following-COVID-19-Shutdown-Update-120620.pdf. 2020:1-24.

31. Centers for Disease Control and Preventions CDC: Guidance for Dental Settings. https://www.cdc.gov/coronavirus/2019-ncov/hcp/dental-settings.html. Centers for Disease Control and Preventions (CDC). 2020:1-14.

32. Australian Dental Association ADA: Decision tree for Patient Management. https://www.ada.org.au/Covid-19-Portal/Dental-Professionals. Australian Dental Association (ADA). 2020:1-48.

\section{Figures}

Figure 1

Hierarchy of controls required for mitigation of COVID-19 spread in a dental clinic environment.

Figure 2

Nosocomial infection risk management framework implemented in a Dental Teaching Facility during the COVID-19 pandemic.

Figure 3

See image above for figure legend

Figure 4

Employed changes in the logistic management of the Dental school and Dental Teaching Hospital during the COVID-19 pandemic.

Figure 5

Clinical training management, session distribution, and workflow before and during the COVID-19 pandemic.

Figure 6

Different stages of COVID-19 pandemic - adapted educational measurements during the highly changing situations. 